OPEN ACCESS

Edited by:

Dongsheng Zhou,

Beijing Institute of Microbiology

and Epidemiology, China

Reviewed by:

Sheng Chen,

Hong Kong Polytechnic University,

China

Aoife Boyd

National University of Ireland, Galway,

Ireland

*Correspondence:

Weihuan Fang,

Molecular Microbiology and Food

Safety Laboratory, Institute

of Preventive Veterinary Medicine, College of Animal Sciences, Zhejiang

University, 388 Yuhangtang Road,

Hangzhou, China

whfang@zju.edu.cn

Specialty section:

This article was submitted to

Food Microbiology,

a section of the journal

Frontiers in Microbiology.

Received: 27 January 2015 Accepted: 12 April 2015

Published: 29 April 2015

Citation:

Shan Y, Fang C, Cheng C, Wang Y, Peng $J$ and Fang $W$ (2015) Immersion infection of germ-free zebrafish with

Listeria monocytogenes induces transient expression of innate immune response genes.

Front. Microbiol. 6:373. doi: 10.3389/fmicb.2015.00373

\section{Immersion infection of germ-free zebrafish with Listeria monocytogenes induces transient expression of innate immune response genes}

\author{
Ying Shan', Chun Fang ${ }^{1}$, Changyong Cheng', Yong Wang ${ }^{2}$, Jinrong Peng ${ }^{2}$ and \\ Weihuan Fang ${ }^{1,3 *}$
}

'Zhejiang Provincial Key Laboratory of Preventive Veterinary Medicine, Institute of Preventive Veterinary Medicine, Zhejiang University, Hangzhou, China, ${ }^{2}$ Key Laboratory for Molecular Animal Nutrition, Ministry of Education, College of Animal Sciences, Zhejiang University, Hangzhou, China, ${ }^{3}$ Molecular Microbiology and Food Safety Laboratory, Institute of Preventive Veterinary Medicine, College of Animal Sciences, Zhejiang University, Hangzhou, China

Zebrafish, Denio rerio, can be an alternative to other classic animal models for human infectious diseases to examine the processes of microbial infections and host-pathogen interactions in vivo because of their small body dimension but large clutch size. We established germ-free zebrafish infection models of Listeria monocytogenes through different routes of infection: oral immersion and injection via yolk sac, brain ventricle and blood island. Immersion of zebrafish larva even with $10^{10} \mathrm{CFU} / \mathrm{mL}$ L. monocytogenes EGDe strain in egg water was unable to cause mortality, but GFP-expressing bacteria in the gut lumen can be observed in frozen sections. Several selected maker genes of the innate immune system, including cyp1a, irg $1 /$, il $1 \mathrm{~b}$, and $\mathrm{mmp9}$, were significantly induced by oral immersion not only with strain EGDe, but also with strain $M 7$ and $L$. innocua, though to a lesser degree $(P<0.01)$. Such induction appears to be transient with peak at $48 \mathrm{~h}$ post-infection, but returned to basal level at $72 \mathrm{~h}$ post-infection. Of the three injection routes, mortality after infection by yolk sac was $80 \%$ in early stage of infection. Few eggs can survive and hatch. Injection into zebrafish embryos via brain ventricle or blood island led to progressive lethal infection. L. mocytogenes EGDe showed steady replication in the fish embryos and was far more pathogenic than strain M7, which is consistent with findings in the murine model. We conclude that zebrafish can serve as susceptible and microscopically visible infection models for L. monocytogenes via different routes and can be applied to further studies on the interactions between bacterial virulence factors and host immune responses.

Keywords: germ-free zebrafish, Listeria monocytogenes, infection model, immersion, innate immune responses

\section{Introduction}

Zebrafish, Denio rerio, as a hybrid animal model between invertebrate and vertebrate, has been widely used for studying human infectious diseases. Zebrafish embryos can be an alternative to other classic animal infection models to examine processes of microbial infections 
and host-pathogen interactions in vivo because of their small body dimension but large clutch size (Tobin et al., 2012). Zebrafish larva has an independent innate immune system. The adaptive immune system is immature until 4-6 weeks post fertilization (Trede et al., 2004). This developing stage during the first 4 week can be employed to study innate immunity to infections by pathogenic organisms.

Several infection techniques have been approached in zebrafish models for over 20 different pathogenic bacterial species (Stockhammer et al., 2009; Li and $\mathrm{Hu}, 2012$ ) as well as several fungal and viral pathogens (Sanders et al., 2003; Chao et al., 2010). The most commonly used method is micro-injection via the blood island near the urogenital opening in the $26 \mathrm{hpf}$ (hours post fertilization) zebrafish. With intravenous infection via blood island, zebrafish is susceptible to almost all bacterial pathogens used so far, including Mycobacterium marinum, Salmonella typhmurium, and Escherichia coli (Prouty et al., 2003; Davis and Ramakrishnan, 2009; Volkman et al., 2010). Other routes of infection include micro-injection into brain ventricle or yolk sac of fish embryos. The brain ventricle is a good site to observe macrophage migration upon intraventricular introduction of pathogens because it is a closed cavity lack of macrophages in the embryonic stage (Davis et al., 2002). Yolk sac injection is suitable for large-scale screening because of its easiness of operation (Carvalho et al., 2011).

Additional advantages of using zebrafish as infection models are that the fish can be readily made germ-free with simple methods (Pham et al., 2008) and that genetic manipulation is relatively easy to generate transgenic lines (Tobin et al., 2012) for some specific experiments to better understand the host-microbe interactions. Two methods were examined for colonizing the developing gut of $5 \mathrm{dpf}$ (days post fertilization) germ-free zebrafish with a defined anaerobic microbial community derived from a single human fecal sample (Toh et al., 2013). Inoculation per os revealed a key role for adhesion in protection by probiotic bacteria in a zebrafish colonization model (Rendueles et al., 2012). These studies suggest that germ-free zebrafish can serve as a useful tool for studying the interaction between specific pathogen and host intestinal mucosa.

Listeria monocytogenes, when orally exposed, can break through the intestinal barrier and cause systemic infections, such as septicemia and meningitis. It has been used as a model intracellular organism in infection biology studies and its pathogenic mechanisms are well illuminated (Cossart, 2007; Stavru et al., 2011). Listeria strains can be isolated from fish (Rodas-Suárez et al., 2006). However, it remains unknown if fish can develop listeriosis by natural way. Adult zebrafish was found less susceptible than mice (Menudier et al., 1996). However, the experiments were conducted at $22^{\circ} \mathrm{C}$, at which temperature Listeria virulence genes are expressed (Johansson et al., 2002). Zebrafish larva at $54 \mathrm{hpf}$ was used for real-time analysis of responses of macrophages to listerial infection by intravenous route (Levraud et al., 2009). The group also indicated that intravenous infection was less lethal than yolk sac infection, and that immersion infection did not induce lethality, possibly indicating failure of the bacterium to cross the intestinal barrier. Edwardsiella tarda, a pathogen natural to aquatic species, presented variable lethality to zebrafish larva upon immersion infection (van Soest et al., 2011). The channel catfish pathogen E. ictaluri was highly pathogenic to zebrafish larvae as shown by high mortality within 3 days after immersion exposure (Rendueles et al., 2012). These studies suggest that pathogenic progression to systemic lethal infection varies with the pathogens used for immersion infection. However, these experiments were conducted on conventional zebrafish where there might be native microbial flora that can interfere with the outcome of infecting pathogens. Also few studies were conducted on germ-free zebrafish for virulence mechanisms of pathogenic bacteria by different routes of infection.

We attempted to use germ-free zebrafish embryos as infection models by multiple routes of inoculation to investigate the pathogenicity of $L$. monocytogenes strains and L. innocua that are known to be of different virulence in mammalian species. Expression of innate immune response genes was examined to study if immersion can induce immune responses in infected fish larvae. We found that immersion infection, though not lethal, can lead to induction of several genes related to innate immunity. Infections by multiple injection routes can clearly differentiate the pathogenic potentials of listerial strains as assessed by hatching rate, surviving larvae and bacterial burden in the body. Such models can be explored to examine the virulence factors of and host responses to pathogenic bacteria other than Listeria.

\section{Materials and Methods}

\section{Bacterial Strains and Culture Condition}

Listeria monocytogenes strain EGDe, strain M7 (a low pathogenic strain isolated from milk in our laboratory; Chen et al., 2010), and L. innuoca strain ATCC33090 were used for infection experiments. To observe the bacteria in vivo, GFP-expressing strains EGDe-gfp, M7-gfp and ATCC-gfp were generated by transforming the respective strains with the recombinant plasmid pFL251 carrying $g f p$ under control of the listerial dlt promoter constructed in our laboratory using the shuttle vector pAM401 (a kind gift from Dr. Nancy E. Freitag) as the backbone. The $d l t$ promoter (in front of dltA, D-alanyl-lipoteichoic acid, of the $d l t$ operon; Fortineau et al., 2000) was PCR amplified from L. monocytogenes EGDe genome. Bacteria were grown in brain heart infusion (BHI, Oxoid, UK) medium at $37^{\circ} \mathrm{C}$ with shaking at $150 \mathrm{rpm}$.

\section{Zebrafish Husbandry and Generation of Germ-Free Embryos}

The zebrafish line AB was provided by Professor JR Peng (Zhejiang University, China). Adult fish were raised in the standard zebrafish unit (Aisheng, Beijing, China) at $28^{\circ} \mathrm{C}$ under a constant light cycle of 14-h on/10-h off. Germ-free embryos were generated with the method previously described (Pham et al., 2008). Natural breeding eggs were collected immediately after hatching and transferred to a sterile dish with sterilized egg water containing antibiotics (ampicillin and kanamycin). Unfertilized embryos were removed timely over the next few days. 


\section{Bacterial Immersion and Injection}

Overnight bacterial cultures were washed with sterile egg water and adjusted to $\mathrm{OD}_{600 \mathrm{~nm}}$ at $0.6\left(10^{9} \mathrm{CFU} / \mathrm{mL}\right)$. Infection was performed by oral immersion and by micro-injections via yolk sac, brain ventricle or blood island, as previously described (Jennifer and Hazel, 2009; Benard et al., 2012). For static immersion, naturally hatched germ-free fish $5 \mathrm{dpf}$ (when mouth and gut are functional) were used. For yolk sac infection, an average of $11 \mathrm{CFU}$ (range $8-14 \mathrm{CFU}$ ) of each listerial strain was micro-injected directly at one-cell stage immediately after fertilization. In models via injection (except for yolk sac infection), $26 \mathrm{hpf}$ fish were dechorionated carefully with ophthalmic forceps and anesthetized. At this stage, they developed into the prim-stage forming closed cavity of brain ventricles and blood island. One nanoliter of each bacterial suspension (mixed with phenol red indicator and contained different concentration of live bacteria depending on the infection routes and experimental purpose) was injected. Effect of different levels of inoculum (EGDe from 11 to $1100 \mathrm{CFU}$ per fish on average) on survival was examined on yet-to-hatch zebrafish by infection via blood island. Intraventricular or intravenous infection was also conducted with fixed inoculum, about $11(8-14)$ or $110(80-140)$ CFU. In all cases, injection with egg water was used as mock infection.

\section{RNA Isolation and Quantitative Reverse Transcription PCR}

Ten embryos of each group were pooled and collected by centrifugation at $12,000 \mathrm{~g}$ for $10 \mathrm{~min}$ and stored at $-80^{\circ} \mathrm{C}$ for later use. Embryos were homogenized in $500 \mu \mathrm{L}$ lysis buffer and total RNA was extracted according to the Uniq10 TRNzol total RNA Extraction and Purification Kit instruction (Tiangen, Beijing, China). DNaseI (Promega, USA) was used to remove residual genomic DNA at $37^{\circ} \mathrm{C}$ for $1 \mathrm{~h}$ before cDNA synthesis. Reverse transcriptase (TOYOBO, Japan) was used for cDNA synthesis. Quantitative reverse transcription PCR was then performed in a $20 \mu \mathrm{l}$ reaction mixture containing SYBR quantitative PCR mix (TOYOBO, Japan) to measure transcriptional levels of immune related genes (cypla, irg1l, il1b, and $m m p 9$ ) with specific primer pairs (Table 1)

TABLE 1 | Quantitative polymerase chain reaction primer sequences used in this study.

\begin{tabular}{lll}
\hline Primers & $\mathbf{5}^{\prime} \mathbf{- 3}^{\prime}$ & Sequence \\
\hline mmp9 & Forward & $5^{\prime}$-CATTAAAGATGCCCTGATGTATCCC \\
& Reverse & $5^{\prime}$-AGTGGTGGTCCGTGGTTGAG \\
il1b & Forward & $5^{\prime}$-GAACAGAATGAAGCACATCAAACC \\
& Reverse & $5^{\prime}$-ACGGCACTGAATCCACCAC \\
cyp1a & Forward & $5^{\prime}$-CCATTCAGACATATCGTAGTATCC \\
& Reverse & $5^{\prime}$-CGCACCAGTTCATCATCATC \\
Irg1 & Forward & $5^{\prime}$-GGTTAGAAGCAAGTCCTC \\
& Reverse & $5^{\prime}$-TGTGTCATCCTCCTCAG \\
F-actin & Forward & $5^{\prime}$-CGAGCTGTCTTCCCATCCA \\
& Reverse & $5^{\prime}$-TCACCAACGTAGCTGTCTITCTG
\end{tabular}

using an iCycler iQ5 real time PCR detection system (BioRad, USA). The housekeeping gene $\beta$-actin was used as internal control for normalization of transcriptional levels of the target genes.

\section{Bacterial Enumeration}

Eight embryos in each group were anesthetized at different time points after intravenous injection. Each embryo was then rinsed and homogenized in $1 \mathrm{~mL}$ sterile egg water. For fish infected by immersion, trunks and intestines were separated by a sterile syringe needle (gage size 26) under the stereo microscope. Five trunks or intestines were pooled and homogenized in $100 \mu \mathrm{L}$ sterile egg water. Serial dilutions in PBS (10 mM, pH 7.4) of the homogenates were plated on PALCAM agar (Listeria selective medium; Luqiao, Beijing, China). The colonies were enumerated after incubation at $37^{\circ} \mathrm{C}$ for $24 \mathrm{~h}$. The results were presented as mean $\log _{10} \mathrm{CFU} \pm \mathrm{SE}$ per five fish.

\section{Cryosection}

Embryos were fixed in $4 \%$ paraformaldehyde for $1 \mathrm{~h}$ at room temperature, then washed three times in sterile egg water (each for $5 \mathrm{~min}$ ) before being embedded in $1.5 \%$ agarose $/ 30 \%$ sucrose, and mounted in a small chamber. The blocks were trimmed to the shape of a pyramid with a surgical blade and equilibrated in $30 \%$ sucrose for at least 1 day at $4^{\circ} \mathrm{C}$. After equilibration, the pyramids were mounted in optimal cutting temperature (O.C.T.) compound (SAKURA) in plastic molds which were then brought onto dry ice for immediate freezing. Prior to cryosectioning, the blocks were mounted on the supporter with O.C.T. compound and equilibrated at $-30^{\circ} \mathrm{C}$ in a pre-chilled microtome (Leica, HM505) for $2 \mathrm{~h}$.

\section{Infection with EGFP-Expressing Listeria and Live Embryo Imaging}

Zebrafish larvae were infected by injection with the EGDe-gfp strain via brain ventricles or blood island and observed microscopically at $6 \mathrm{hpi}$ (brain infection) or at 6, 24, and $48 \mathrm{hpi}$ (intravenous). The GFP-expressing bacteria were monitored and photographed in vivo by laser confocal microscope IX81-FV1000 (Olympus, Japan).

\section{Confirmation to the Relevant Regulatory Standards}

All animal experiments in this study were approved by the Laboratory Animal Management Committee of Zhejiang University (Approval No. 2013038).

\section{Results}

\section{Oral Infection Did Not Cause Death, but Induced Transcription of Innate Immunity Related Genes}

In order to mimic the natural route of infection through the digestive tract in mammalian species, we performed oral infection of germ-free fish by static immersion of different concentration of bacterial suspension of the EGDe strain. However, there 
was no death even with $10^{10} \mathrm{CFU} / \mathrm{mL}$ in egg water during the 10-days observation period. To test if the bacteria enter into the gut, we imaged sections of zebrafish 24,48 , and 72 dpi by confocal microscope and found that there were significant amount of EGDe-gfp bacteria in the gut lumen 24 and 48 hpi but not in any other tissues (Figure 1) while M7-gfp or ATCC-gfp can not be observed. In 72 hpi fish sections, bacteria was no longer observed in gut lumen.

To examine immune responses to such non-lethal infection, the transcriptional levels of genes related to innate immunity Cyp1a, Irg1l, Illb, and Mmp9 were analyzed from fish at 24, 48 , and $72 \mathrm{hpi}$. We found that these genes were significantly induced at $48 \mathrm{hpi}$ but returned to the normal level at $72 \mathrm{hpi}$ (Figure 2). Highest induction was observed in strain EGDe infected fish. These results were consistent with the findings by plate counting and confocal imaging that bacterial burden in EGDe exposed fish was much higher than that in M7 or L. innocua exposed fish both in the trunk and in intestine (Figure 3).

\section{Hatchability After Yolk Sac Injection was Reduced and the Reduction Varied with Strains}

Infection with EGDe caused higher mortality (82\%) with few eggs survived and hatched than the strain M7 (20\%, $P<0.005$; Figure 4) previously shown as low pathogenic in the mouse model (Chen et al., 2010). There was virtually no difference of hatchability between the L. innuoca and mock groups (88 vs. 90\%), indicating the non-pathogenic nature of this listerial species.

\section{Survival Rate Differed Among Strains After Brain Ventricle Injection}

Brain ventricle inoculation led to progressive lethal infection with two L. monocytogenes strains (Figure 5). However, EGDe showed more significant death than M7 at day 5 post-infection (dpi; 90 vs. 55\%, $P<0.05$ ). No further death was seen with M7, while there was no survival with EGDe at $10 \mathrm{dpi}$. Infection with L. innocua did not
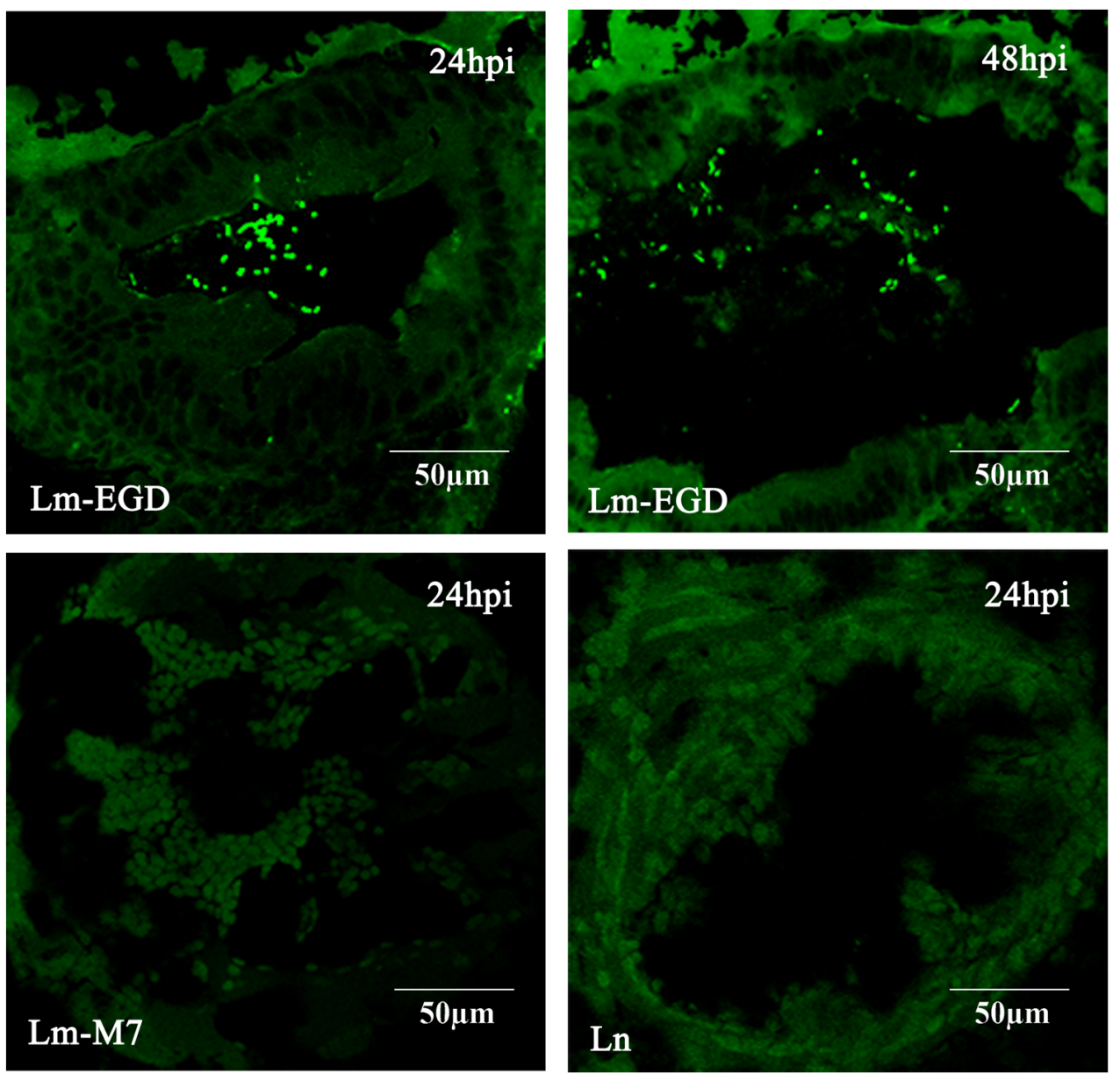

FIGURE 1|Bacterial distribution in representative frozen sections of germ-free zebrafish infected at 5 dpf by $24 \mathrm{~h}$ of immersion with GFP-expressing listerial strain. Images were taken with the confocal microscope in samples taken at 24 and
$48 \mathrm{~h}$ post infection (hpi; five fish/strain at each time point). Lm-EGD and Lm-M7 stand for L. monocytogenes strains EGDe and $M 7$ respectively; and $L n$, for $L$. innocua. Same abbreviations are used for the following figures. 


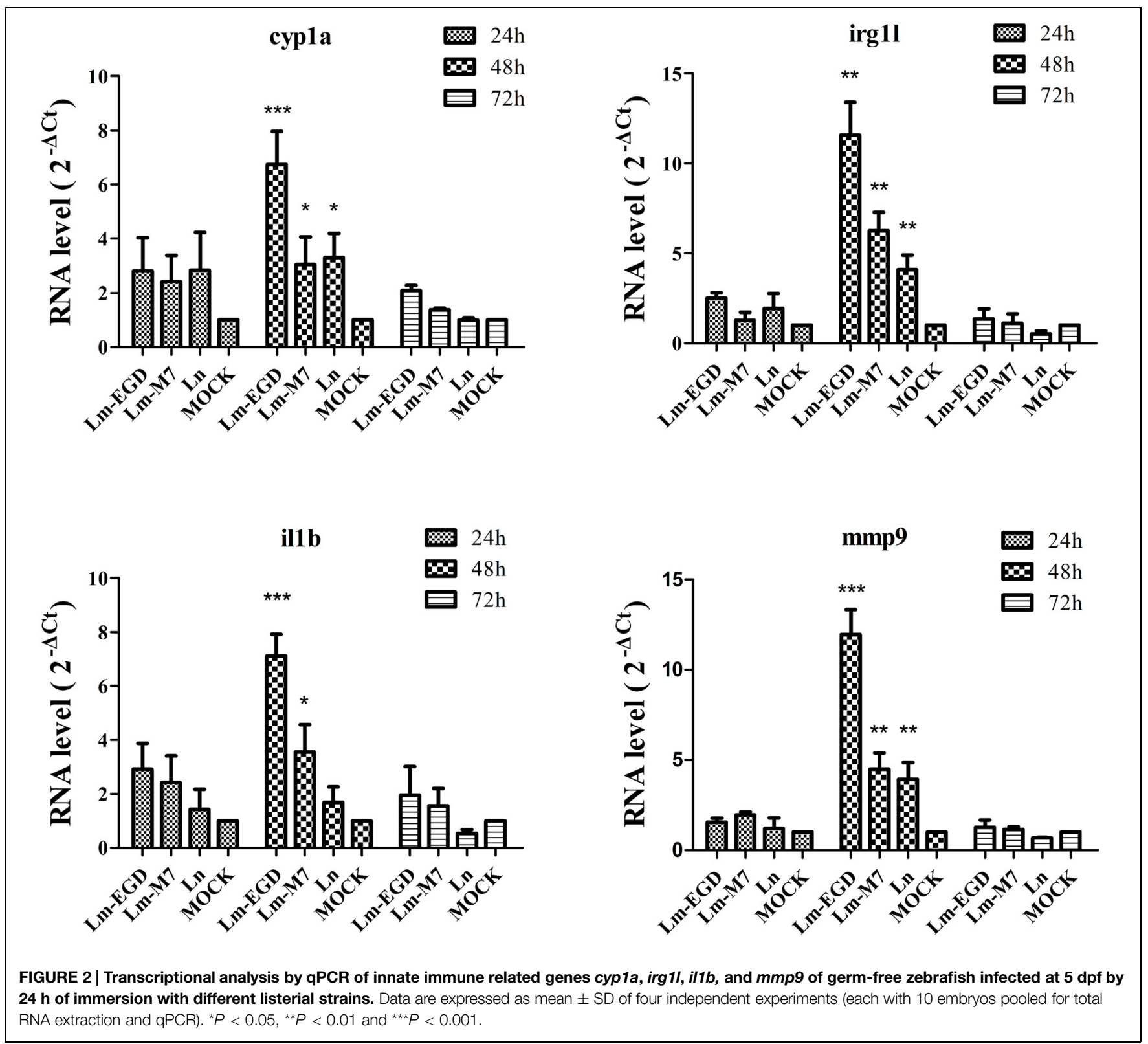

cause significant death, as compared with the mock infection. Macrophages at the infected site (Figure 6A, white box) were observed at 6hpi. Confocal microscopic examination showed that macrophages migrated to the brain ventricle (Figure 6B) and contained engulfed/attached bacteria (Figures 6C-F).

\section{Survival Rate was Dose-Dependent and Varied with Strains Upon Intravenous Infection}

Figure 7A shows that EGDe infection via the blood island resulted in progressive death with $100 \%$ mortality from day 2 to 7 , depending on the inoculum level from 11 to $1100 \mathrm{CFU}$ per fish. At similar inoculum levels (110 CFU per fish, ranges from 80 to $140 \mathrm{CFU}$ ), EGDe was far more pathogenic than M7 at $4 \mathrm{dpi}$ (survival rate: 30 vs. $85 \%, P<0.001)$ and at $8 \mathrm{dpi}(0$ vs. 55\%, $P<0.001)$. L. innocua caused marginal death. By bacterial enumeration, we found that that there was initial growth of all inoculated strains from 6 to 24 hpi with EGDe having the highest bacterial load (Figure 7C). While the M7 strain and nonpathogenic L. innocua did not show further growth, the EGDe strain continued its growth at 48 hpi when fish death began (Figures 7A-C). To observe the bacterial load in vivo, we also infected the fish with GFP-expressing EGDe. Figure 8 shows that EGDe strain had substantial proliferation in the fish over time. The bacteria could be randomly observed at the injection site at 6 hpi (Figures 8D,G), and then distributed in trunk and cardinal vessels at $24 \mathrm{hpi}$ (Figures 8E,H). Substantial bacterial proliferation was seen in the trunk (Figure 8F) or in cerebral and retinal vessels (Figure 8I) at $48 \mathrm{hpi}$. 
Trunk

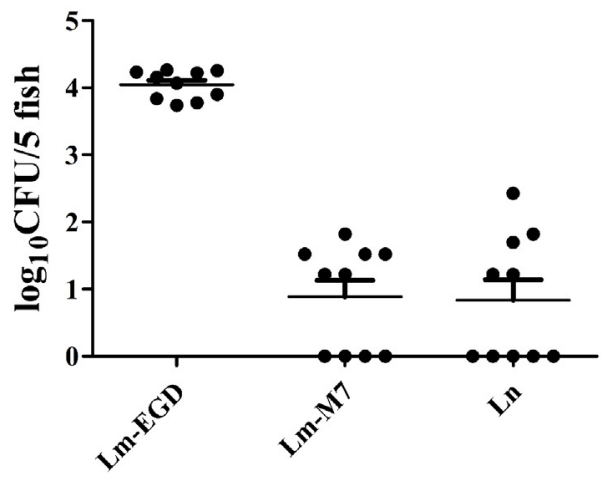

FIGURE 3|Bacterial burden in the body trunk and intestine of $L$. monocytogenes EGDe infected germ-free fish was much higher than that of the strain $\mathbf{M 7}$ or $\boldsymbol{L}$. innocua infected ones. The fish (50 per strain) were immersion-infected at $5 \mathrm{dpf}$ and sacrificed at $24 \mathrm{hpi}$. The body trunk and intestine samples of each strain were
Intestine

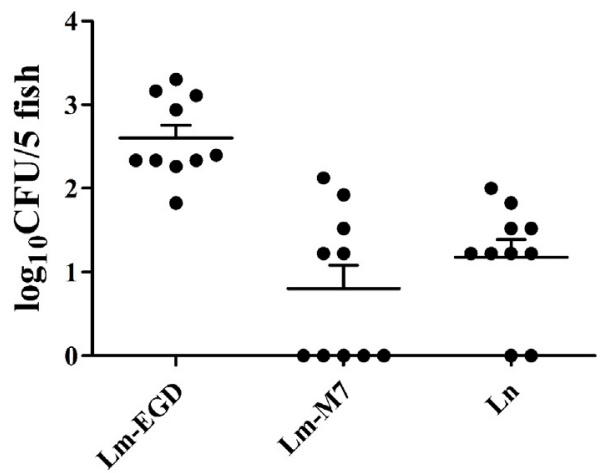

divided into 10 subgroups, each having five fish larvae to be homogenized and resuspended in $100 \mu$ of PBS, from which a volume of $10-\mu$ l suspension was spotted in triplicate on the PALCAM agar plates for bacterial enumeration. Data were expressed as $\log _{10}$ CFU per five fish.

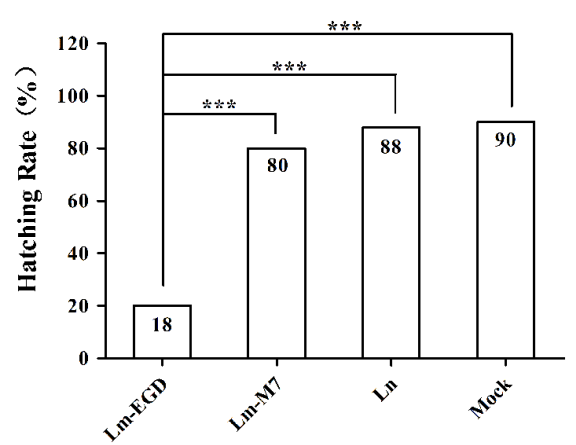

FIGURE 4 | Hatching rate of zebrafish eggs infected with different listerial strains ( $10 \mathrm{CFU}$ per fish, $n=50$ ) via yolk sac injection. ${ }^{\star \star \star} P<0.001$

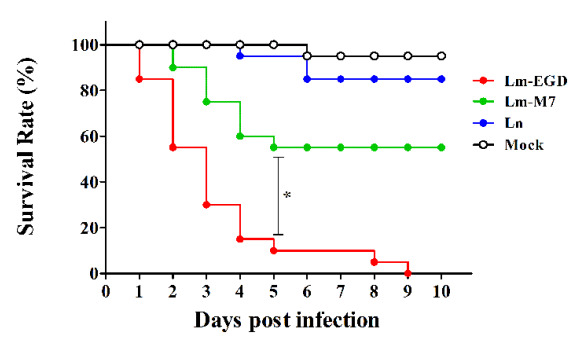

FIGURE 5 | Percent survival of 26 hpf germ-free zebrafish embryos $(n=20)$ infected with different listerial strains (100 CFU per fish) via brain ventricle injection. ${ }^{\star} P<0.05$.

\section{Discussion}

Stages of embryonic development of zebrafish have been well described since 1990s (Kimmel et al., 1995). The small transparent zebrafish embryos have been utilized as an alternative to mammalian animal models for studies of microbial infection and host responses (Cui et al., 2011; Meijer and Spaink, 2011; Novoa and Figueras, 2012). Previous work mostly focused on infections of conventional zebrafish embryos by individual routes of inoculation of their target microorganisms (Van Der Sar et al., 2003; Levraud et al., 2009; Volkman et al., 2010). A growing number of studies used the germ-free zebrafish to examine the immune responses to infections (Rendueles et al., 2012; Toh et al., 2013).

To ensure the sterility of germ-free zebrafish embryos we generated according to one of the methods described elsewhere (Pham et al., 2008), we monitored the total bacterial count in egg water and fish embryos on BHI agar up to $5 \mathrm{dpf}$. No bacterial colonies were seen. To further ensure that zebrafish embryos generated in our system were developing without the activation of innate immunity due to possible microbial colonization, we tested the expression of several Toll-like receptors in $5 \mathrm{dpf}$ zebrafish embryos. Homogenized samples of germfree and conventionally raised zebrafish were sacrificed for RNA extraction and RT-PCR. Five tlr genes (tlr1, tlr2, tlr 3 , tlr $4 b$, and $t l r 5 b$ ) were selected from the list of 23 predicted zebrafish tlr genes (Meijer et al., 2004). Their transcriptional levels were barely detectable in zebrafish raised in the germ-free system, but highly induced in conventionally raised zebrafish (data not shown).

Initially we hypothesized that conventional zebrafish procolonized with commensal bacteria might render the fish resistant to immersion infection with pathogenic bacteria such as $L$. monocytogenes. We attempted to infect germ-free zebrafish larva to mimic the natural route of listeria infection through the digestive gut using the strains of different pathogenicity already known in other animal models (Schlech et al., 1993). Immersion inoculation to germ-free zebrafish at $5 \mathrm{dpf}$, a time with their mouth open and gut functional, did not cause any lethal infection even with the inoculum of the pathogenic L. monocytegenes EGDe as high as $10^{10} \mathrm{CFU} / \mathrm{ml}$ egg water. Lack of lethality was also seen in immersion infection with $L$. monocytegenes (Levraud et al., 

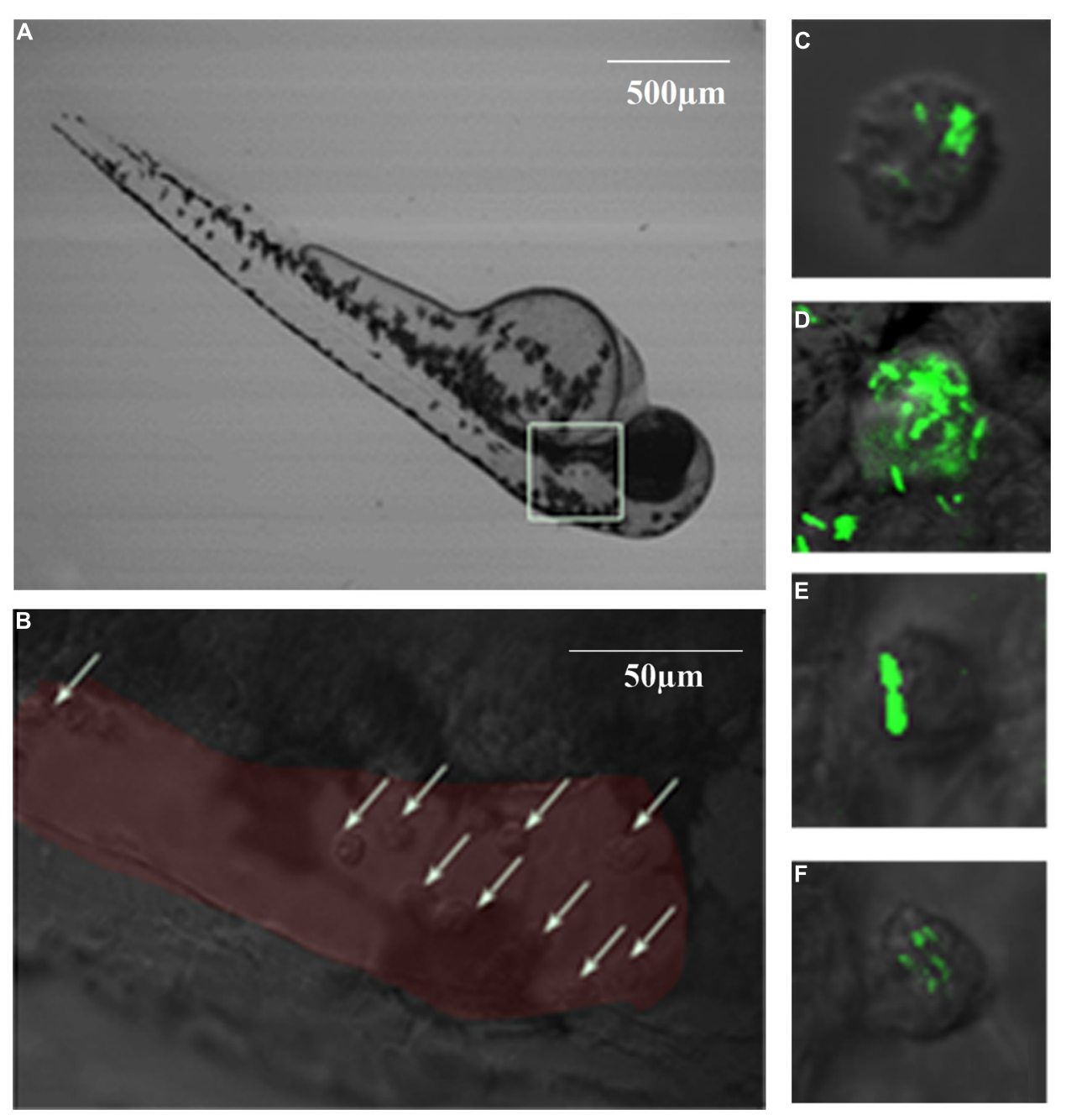

FIGURE 6 | Phagocytosis and bacterial proliferation in macrophages in 6 hpi germ-free zebrafish after brain ventricle injection with $L$. monocytogenes EGDe. (A): Injection site at low magnification; (B): High magnification of the injection site (red area) showing recruitment of macrophages (white arrows) to the brain ventricle in response to bacterial infection; (C-F): GFP-expressing bacteria were engulfed by macrophages.
2009) or E. tarda (van Soest et al., 2011) using conventional fish, while immersion infection of the conventional zebrafish with E. ictaluri led to high mortality (Rendueles et al., 2012). These results seem to indicate that the virulence determinants of the pathogens might be the key players in breaking through the gut barrier and cause systemic infection, and that deprivation of commensal microbes and lack of pre-stimulated immune responses do not predispose the fish to lethal infection.

To study if immersion could induce immune responses in infected fish larvae, several immune related genes were examined. Cyp1a is involved in intestinal epithelial detoxication and regulated by Toll-like receptor 2 in mice (Yamazaki et al., 2002; Do et al., 2012). Irg1l is homologous to mammalian irg1 (immunoresponsive gene 1) which is expressed by macrophages and can be induced by proinflammatory cytokines (Degrandi et al., 2009). Illb, a member of the interleukin 1 family of cytokines, is an important mediator of the inflammatory response
(Barksby et al., 2007; Dinarello, 2009). Mmp9 is a member of the matrix metalloproteinase (MMP) family that is important for remodeling of the extracellular matrix (Yoong et al., 2007). Oral immersion, though not lethal, induced expression of selected genes that peaked at 48 hpi but returned to normal at 72 hpi. It is clear that the pathogenic L. monocytegenes EGDe strain caused highest induction with nearly twice the transcriptional level of the low-pathogenic strain M7 and $L$. innocua. The latter two strains had similar induction levels in general, but significantly higher than the un-inoculated control. These findings suggest that stimulation of the innate immune responses was closely related to the virulence of the strains used.

Significant induction of $\operatorname{Irg} 1 \mathrm{l}, \operatorname{mmp} 9$, and $i l 1 b$ at $24 \mathrm{hpi}$ was also seen from 25-hpf conventional fish immersion-infected with E. tarda (van Soest et al., 2011). However, we did not found such significant induction at this time point. Robust 
A

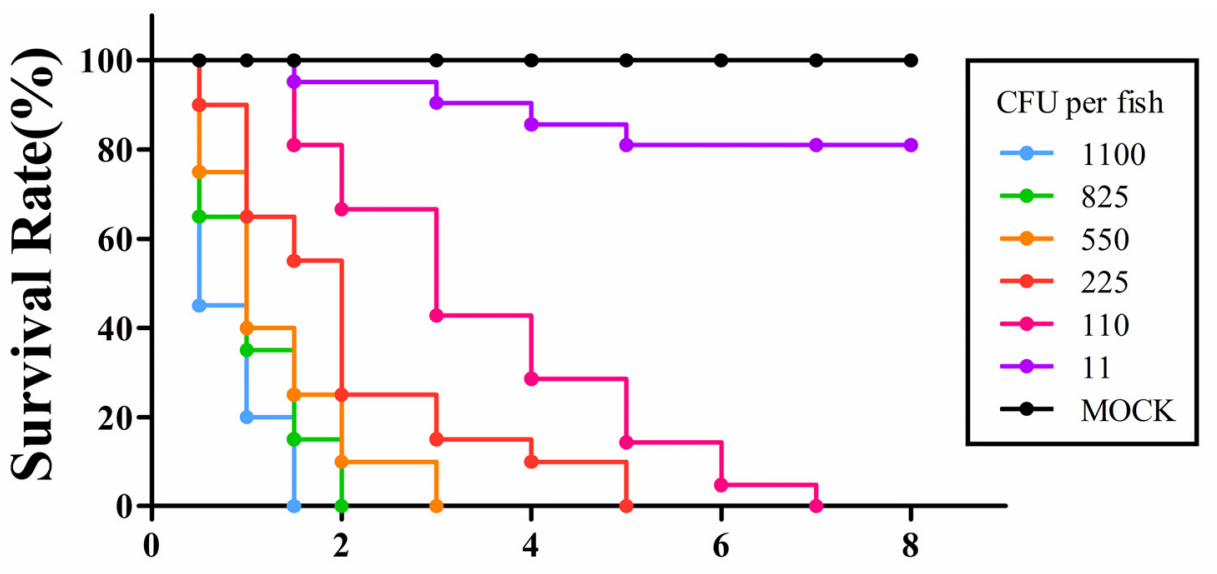

Days post infection

B

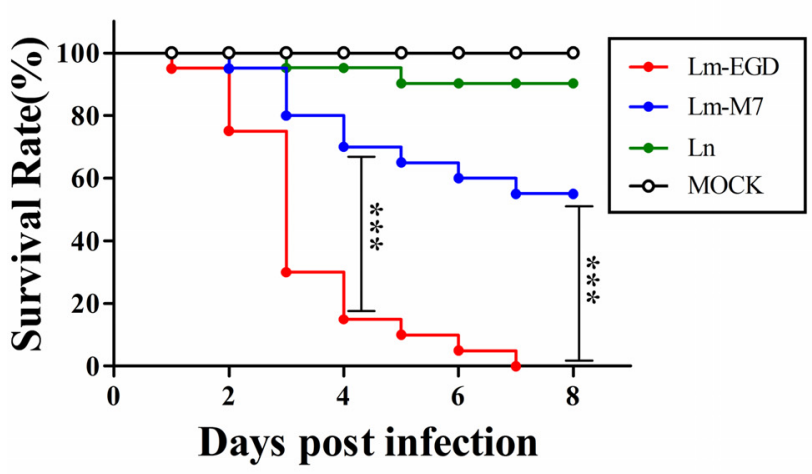

C

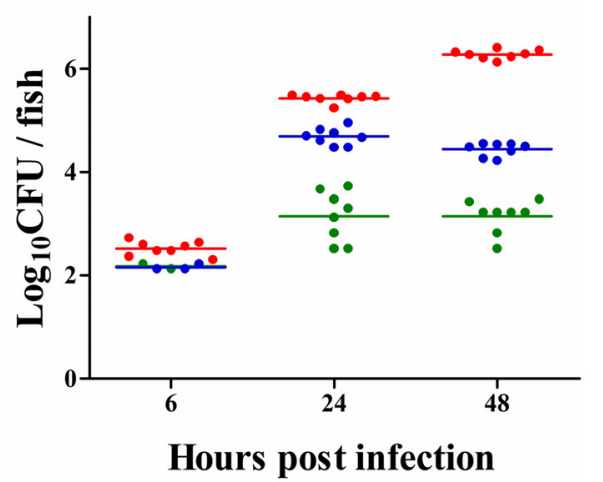

FIGURE 7 | Infection of germ-free zebrafish embryos, via intravenous injection, with graded inocula of $L$. monocytogenes EGDe (A, \% survival with $n=20$ ), or with similar inocula (80-140 CFU on average) of $L$. monocytogenes strains $\mathrm{EGDe}$ and $\mathrm{M7}$ as well as $\mathrm{L}$. innocua $(\mathrm{B}, \%$ survival with $n=20$; and $C$, bacterial proliferation with $n=8$; and same

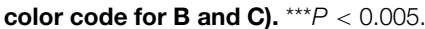

induction of IL-1 $\beta$ and TNF- $\alpha$ was observed as early as 2 hpi (TNF- $\alpha)$ or 4 hpi (IL-1 $\beta$ ) in conventional zebrafish larvae immersion-infected with E. tarda at $24 \mathrm{hpf}$ (Pressley et al., 2005). Besides different pathogenic species used, the microbial status may contribute to distinct responses between germfree and conventional fish. Colonization of gnotobiotic $3 \mathrm{dpf}$ fish larvae with conventional zebrafish microbeta induces NF$\kappa \mathrm{B}$ pathway activation in a dynamic temporal pattern with peak at $6 \mathrm{dpf}$ (72 hpi; Kanther et al., 2011), about 24-h later than what we saw (48 hpi). Thus, it is tempting to speculate that priming of the fish with indigenous commensals at early age could facilitate the innate immune responses to invading pathogens. Bates et al. (2006) found that absence of microbiota during developmental stages of zebrafish was found to have arrested differentiation and altered function of macrophages.

Similar to our finding that expression of the innate immune genes returned to normal at $72 \mathrm{hpi}$, Pressley et al. (2005) observed even quicker decline of IL-1 $\beta$ at 12 hpi (from its peak at $4 \mathrm{hpi}$ ) in E. tarda infected fish by immersion. Such phenomena may indicate clearance of invaded pathogens by the macrophages. Herbomel et al have revealed that the embryonic macrophages of zebrafish are different from the adult, capable of proliferation and able to clear bacterial infection efficiently (Herbomel et al., 1999). Macrophages of zebrafish larvae could also control early $M$. marinum infection (Clay et al., 2007).

With injection inoculation, we found that the EGDe strain was far more pathogenic than the strain M7, as shown by significantly lower hatchability (yolk sac infection) and higher mortality (both intraventricular and intravenous infections). L. innocua strain did not show apparent lethality in all these infection routes. These results were consistent with murine models that shows the virulent nature of the EGDe strain (Brosch et al., 1993; Bécavin et al., 2014) and the low-pathogenicity of the strain M7 (Chen et al., 2010). This is in general agreement with major findings in a conventional zebrafish model showing that L. monocytegenes strain EGDe was more pathogenic than 


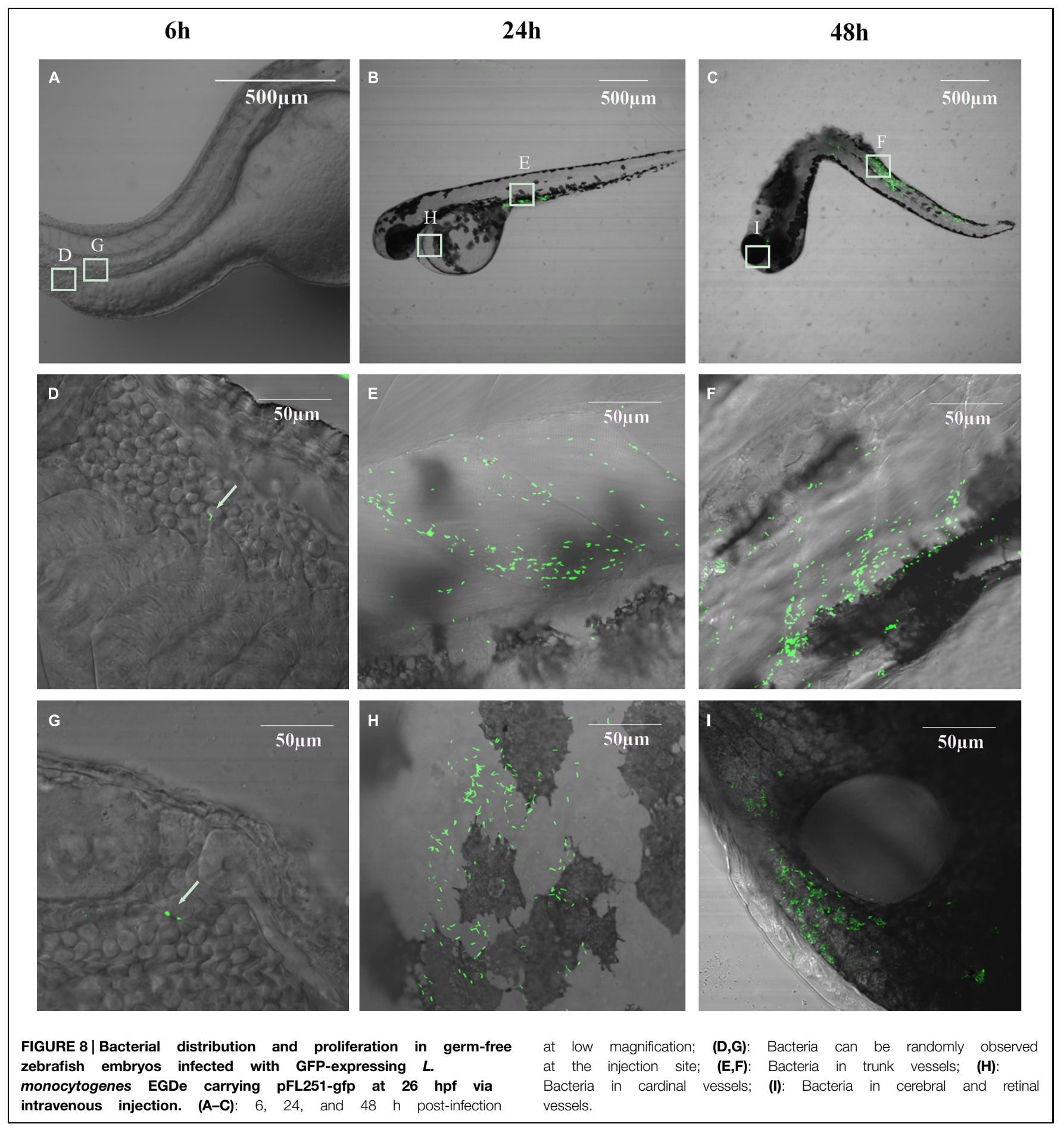

L. innocua (Levraud et al., 2009). However, the germ-free fish larvae were more susceptible to EGDe infection since the bacterial number causing $100 \%$ mortality within 2 dpi in our study was $\sim 10^{3}$ CFU, about 1 log less than that reported by Levraud et al. (2009).

The brain ventricle is a closed cavity which contains zero to two macrophages in zebrafish larva (Davis et al., 2002). Migration of macrophages into this cavity has been observed in several previous work examining the innate immune responses to infection (Herbomel et al., 1999; Clay et al., 2007). Besides the progressive lethality caused by brain ventricle injection with EGDe, we also observed macrophage migration to the brain ventricle and bacterial proliferation within the macrophages. This route of infection in the $26 \mathrm{hpf}$ zebrafish embryos, a developmental period not hatched and without pigment formation, can serve as a good transparent model for 
visual observation of trafficking of phagocytic cells through the blood-brain barrier if the transgenic fish line (Li et al., 2012) is used.

In summary, our findings clearly indicate that immersion infection of the germ-free fish larvae induces distinct innate immune responses to Listeria strains of different pathogenicity. Since there is clear distinction of responses to infection between germ-free and conventional fish, possibly because of the confounding effects from the indigenous commensals in the conventional fish, the germ-free fish are preferred to examine immune responses to infection by pathogenic Listeria or other pathogens, such as trafficking of phagocytes and induction of inflammatory cytokines or genes related to innate immunity. Such work would help elucidate the molecular determinants of the host immune responses to infection and the virulence factors of the pathogen in initiating successful infection, when combined with readily

\section{References}

Barksby, H. E., Lea, S. R., Preshaw, P. M., and Taylor, J. J. (2007). The expanding family of interleukin-1 cytokines and their role in destructive inflammatory disorders. Clin. Exp. Immunol. 149, 217-225. doi: 10.1111/j.13652249.2007.03441.x

Bates, J. M., Mittge, E., Kuhlman, J., Baden, K. N., Cheesman, S. E., and Guillemin, K. (2006). Distinct signals from the microbiota promote different aspects of zebrafish gut differentiation. Dev. Biol. 297, 374-386. doi: 10.1016/j.ydbio.2006.05.006

Bécavin, C., Bouchier, C., Lechat, P., Archambaud, C., Creno, S., Gouin, E., et al. (2014). Comparison of widely used Listeria Monocytogenes strains egd, 10403s, and egd-E highlights genomic differences underlying variations in pathogenicity. mBio. 5:e00969-14. doi: 10.1128/mBio.00969-14

Benard, E. L., van der Sar, A. M., Ellett, F., Lieschke, G. J., Spaink, H. P., and Meijer, A. H. (2012). Infection of zebrafish embryos with intracellular bacterial pathogens. J. Vis. Exp. 61:3781. doi: 10.3791/3781

Brosch, R., Catimel, B., Milon, G., Buchrieser, C., Vindel, E., and Rocourt, J. (1993). Virulence heterogeneity of Listeria Monocytogenes strains from various sources (food, human, animal) in immunocompetent mice and its association with typing characteristics. J. Food Prot. 56, 297-301.

Carvalho, R., de Sonneville, J., Stockhammer, O. W., Savage, N. D., Veneman, W. J., Ottenhoff, T. H., et al. (2011). A high-throughput screen for tuberculosis progression. PLoS ONE 6:e16779. doi: 10.1371/journal.pone.0016779.

Chao, C.-C., Hsu, P.-C., Jen, C.-F., Chen, I.-H., Wang, C.-H.,Chan, H.-C., et al. (2010). Zebrafish as a model host for Candida Albicans Infection. Infect. Immun. 78, 2512-2521. doi: 10.1128/IAI.01293-09

Chen, J., Chen, Q., Jiang, J., Hu, H., Ye, J., and Fang, W. (2010). Serovar 4 b complex predominates among Listeria monocytogenes isolates from imported aquatic products in China. Foodborne Pathog. Dis. 7, 31-41. doi: 10.1089/fpd.2009.0353

Clay, H., Davis, J., Beery, D., Huttenlocher, A., Lyons, S. E., and Ramakrishnan, L. (2007). Dichotomous role of the macrophage in early Mycobacterium marinum infection of the zebrafish. Cell Host Microbe 2, 29-39. doi: 10.1016/j.chom.2007.06.004

Cossart, P. (2007). Listeriology (1926-2007): the rise of a model pathogen. Microbes Infect. 9, 1143-1146. doi: 10.1016/j.micinf.2007.05.001

Cui, C., Benard, E. L., Kanwal, Z., Stockhammer, O. W., van der Vaart, M., Zakrzewska, A., et al. (2011). Infectious disease modeling and innate immune function in zebrafish embryos. Methods Cell Biol. 105, 273-308. doi: 10.1016/B978-0-12-381320-6.00012-6

Davis, J., Clay, H., Lewis, J. L., Ghori, N., Herbomel, P., and Ramakrishnan, L. (2002). Real-yime visualization of mycobacterium-macrophage interactions leading to initiation of granuloma formation in Zebrafish embryos. Immunity 17, 693-702. doi: 10.1016/S1074-7613(02)00475-2

Davis, J., and Ramakrishnan, L. (2009). The Role of the granuloma in expansion and dissemination of early Tuberculous Infection. Cell 136, 37-49. doi: 10.1016/j.cell.2008.11.014 available genetic manipulation technologies on both zebrafish and pathogens of interests.

\section{Author Contributions}

WF, JP, and YS conceived and designed the experiments. YS, CF, $\mathrm{CC}$, and YW performed the experiments. WF, JP, and YS analyzed the data. YS and WH wrote the paper.

\section{Acknowledgments}

This work is funded in part by Zhejiang Provincial Department of Science and Technology (2014C02003) and National Natural Science Foundation of China (30870068).

Degrandi, D., Hoffmann, R., Beuter-Gunia, C., and Pfeffer, K. (2009). The proinflammatory cytokine-induced Irg1 protein associates with mitochondria. J. Interferon Cytokine Res. 29, 55-68. doi: 10.1089/jir.2008.0013

Dinarello, C. A. (2009). Immunological and inflammatory functions of the interleukin-1 family. Annu. Rev. Immunol. 27, 519-550. doi: 10.1146/annurev.immunol.021908.132612

Do, K. N., Fink, L. N., Jensen, T. E., Gautier, L., and Parlesak, A. (2012). Tlr2 controls intestinal carcinogen detoxication by Cypla1. PLoS ONE 7:e32309. doi: 10.1371/journal.pone.0032309

Fortineau, N., Trieu-Cuot, P., Gaillot, O., Pellegrini, E., Berche, P., and Gaillard, J. L. (2000). Optimization of green fluorescent protein expression vectors for in vitro and in vivo detection of Listeria monocytogenes. Res. Microbiol. 151, 353-360. doi: 10.1016/S0923-2508(00)00158-3

Herbomel, P., Thisse, B., and Thisse, C. (1999). Ontogeny and behaviour of early macrophages in the zebrafish embryo. Development 126, 3735-3745.

Jennifer H, G., and Hazel, S. (2009). Zebrafish brain ventricle injection. J. Vis. Exp. 26:1218. doi: $10.3791 / 1218$

Johansson, J., Mandin, P., Renzoni, A., Chiaruttini, C., Springer, M., and Cossart, P. (2002). An Rna thermosensor controls expression of virulence genes in Listeria monocytogenes. Cell 110, 551-561. doi: 10.1016/S0092-8674(02)00905-4

Kanther, M., Sun, X., Mühlbauer, M., Mackey, L. C., Flynn, E. J., Bagnat, M., et al. (2011). Microbial colonization induces dynamic temporal and spatial patterns of Nf-Kb activation in the zebrafish digestive tract. Gastroenterology 141, 197-207. doi: 10.1053/j.gastro.2011.03.042

Kimmel, C. B., Ballard, W. W., Kimmel, S. R., Ullmann, B., and Schilling, T. F. (1995). Stages of embryonic development of the zebrafish. Dev. Dyn. 203, 253-310. doi: 10.1002/aja.1002030302

Levraud, J.-P., Disson, O., Kissa, K., Bonne, I., Cossart, P., Herbomel, P., et al. (2009). Real-time observation of Listeria monocytogenes-Phagocyte interactions in living zebrafish larvae. Infect. Immun. 77, 3651-3660. doi: 10.1128/IAI.00408-09

Li, L., Yan, B., Shi, Y.-Q., Zhang, W.-Q., and Wen, Z.-L. (2012). Live imaging reveals differing roles of macrophages and neutrophils during zebrafish tail fin regeneration. J. Biol. Chem. 287, 25353-25360. doi: 10.1074/jbc.M112.349126

Li, Y., and Hu, B. (2012). Establishment of multi-site infection model in zebrafish larvae for studying Staphylococcus aureus infectious disease. J. Genet. Genomics 39, 521-534. doi: 10.1016/j.jgg.2012.07.006

Meijer, A. H., Gabby Krens, S. F., Medina Rodriguez, I. A., He, S., Bitter, W., Ewa Snaar-Jagalska, B., et al. (2004). Expression analysis of the toll-like receptor and tir domain adaptor families of zebrafish. Mol. Immunol. 40, 773-783. doi: 10.1016/j.molimm.2003.10.003

Meijer, A. H., and Spaink, H. P. (2011). Host-pathogen interactions made transparent with the zebrafish model. Curr. Drug Targets 12, 1000-1017. doi: 10.2174/138945011795677809

Menudier, A., Rougier, F. P., and Bosgiraud, C. (1996). Comparative virulence between different strains of Listeria in Zebrafish (Brachydanio Rerio) and Mice. Pathol. Biol. 44, 783-789. 
Novoa, B., and Figueras, A. (2012). "Zebrafish: model for the study of inflammation, and the innate immune response to infectious diseases," in Current Topics in Innate Immunity II, eds J. D. Lambris and G. Hajishengallis (New York: Springer), 253-275.

Pham, L. N., Kanther, M., Semova, I., and Rawls, J. F. (2008). Methods for generating and colonizing gnotobiotic Zebrafish. Nat. Protoc. 3, 1862-1875. doi: 10.1038/nprot.2008.186

Pressley, M. E., Phelan, P. E., Witten, P. E., Mellon, M. T., and Kim, C. H. (2005). Pathogenesis and inflammatory response to Edwardsiella tarda infection in the zebrafish. Dev. Comp. Immunol. 29, 501-513. doi: 10.1016/j.dci.2004.10.007

Prouty, M. G., Correa, N. E., Barker, L. P., Jagadeeswaran, P., and Klose, K. E. (2003). Zebrafish-Mycobacterium marinum model for mycobacterial pathogenesis. FEMS Microbiol. Lett. 225, 177-182. doi: 10.1016/S0378-1097(03)00446-4

Rendueles, O., Ferrières, L., Frétaud, M., Bégaud, E., Herbomel, P., Levraud, J. P., et al. (2012). A new Zebrafish model of oro-intestinal pathogen colonization reveals a key role for adhesion in protection by probiotic bacteria. PLoS Pathog. J. 8:e1002815. doi: 10.1371/journal.ppat.1002815

Rodas-Suárez, O. R., Flores-Pedroche, J. F., Betancourt-Rule, J. M., QuiñonesRamírez, E. I., and Vázquez-Salinas, C. (2006). Occurrence and antibiotic sensitivity of Listeria monocytogenes strains isolated from oysters, fish, and estuarine water. Appl. Environ. Microbiol. 72, 7410-7412. doi: 10.1128/AEM.00956-06

Sanders, G. E., Batts, W. N., and Winton, J. R. (2003). Susceptibility of zebrafish (Danio rerio) to a model pathogen, spring viremia of carp virus. Comp. Med. 53, 514-521.

Schlech, W. F. III, Chase, D. P., and Badley, A. (1993). A model of food-borne Listeria Monocytogenes infection in the sprague-dawley rat using gastric inoculation: development and effect of gastric acidity on infective dose. Int. J. Food Microbiol. 18, 15-24. doi: 10.1016/0168-1605(93)90003-Y

Stavru, F., Archambaud, C., and Cossart, P. (2011). Cell biology and immunology of Listeria monocytogenes infections: novel insights. Immunol. Rev. 240, 160-184. doi: 10.1111/j.1600-065X.2010.00993.x

Stockhammer, O. W., Zakrzewska, A., Hegedûs, Z., Spaink, H. P., and Meijer, A. H. (2009). Transcriptome profiling and functional analyses of the zebrafish embryonic innate immune response to Salmonella Infection. J. Immunol. 182, 5641-5653. doi: 10.4049/jimmunol.0900082

Tobin, D. M., May, R. C., and Wheeler, R. T. (2012). Zebrafish: a see-through host and a fluorescent toolbox to probe host-pathogen interaction. PLoS Pathog. J. 8:e1002349. doi: 10.1371/journal.ppat.1002349
Toh, M. C., Goodyear, M., Daigneault, M., Allen-Vercoe, E., and Van Raay,T. J. (2013). Colonizing the embryonic Zebrafish gut with anaerobic bacteria derived from the human gastrointestinal tract. Zebrafish 10, 194-198. doi: 10.1089/zeb.2012.0814

Trede, N. S., Langenau, D. M., Traver, D., Look, A. T., and Zon, L. I. (2004). The use of zebrafish to understand immunity. Immunity 20, 367-379. doi: 10.1016/S1074-7613(04)00084-6

Van Der Sar, A. M., Musters, R. J., Van Eeden, F. J., Appelmelk, B. J., Vandenbroucke-Grauls, C. M., and Bitter, W. (2003). Zebrafish embryos as a model host for the real time analysis of Salmonella typhimurium infections. Cell. Microbiol. 5, 601-611. doi: 10.1046/j.1462-5822.2003. 00303. $\mathrm{x}$

van Soest, J. J., Stockhammer, O. W., Ordas, A., Bloemberg, G. V., Spaink, H. P., and Meijer, A. H. (2011). Comparison of static immersion and intravenous injection systems for exposure of zebrafish embryos to the natural pathogen Edwardsiella tarda. BMC Immunol. 12:58. doi: 10.1186/1471-2172-12-58

Volkman, H. E., Pozos, T. C., Zheng, J., Davis, J. M., Rawls, J. F., and Ramakrishnan, L. (2010). Tuberculous granuloma induction via interaction of a bacterial secreted protein with host epithelium. Science 327, 466-469. doi: 10.1126/science.1179663

Yamazaki, K., Teraoka, H., Dong, W., Stegeman, J. J., and Hiraga, T. (2002). Cdna cloning and expressions of cytochrome P450 1a in zebrafish embryos. J. Vet. Med. Sci. 64, 829-833. doi: 10.1292/jvms.64.829

Yoong, S., O'Connell, B., Soanes, A., Crowhurst, M. O., Lieschke, G. J., and Ward, A. C. (2007). Characterization of the zebrafish matrix metalloproteinase 9 gene and its developmental expression pattern. Gene Expr. Patterns 7, 39-46. doi: 10.1016/j.modgep.2006.05.005

Conflict of Interest Statement: The authors declare that the research was conducted in the absence of any commercial or financial relationships that could be construed as a potential conflict of interest.

Copyright (c) 2015 Shan, Fang, Cheng, Wang, Peng and Fang. This is an open-access article distributed under the terms of the Creative Commons Attribution License (CC BY). The use, distribution or reproduction in other forums is permitted, provided the original author(s) or licensor are credited and that the original publication in this journal is cited, in accordance with accepted academic practice. No use, distribution or reproduction is permitted which does not comply with these terms. 